\title{
SMOOTHNESS OF $\psi$-DIRECT SUMS OF BANACH SPACES
}

\author{
Ken-ICHI Mitani, SAToru Oshiro AND Kichi-SuKe SAito
}

Abstract. Let $X_{1}, X_{2}, \cdots, X_{n}$ be Banach spaces and $\psi$ a continuous convex function with some appropriate conditions on a certain convex subset of $\mathbb{R}^{n}$. Let $\left(X_{1} \oplus X_{2} \oplus \cdots \oplus X_{n}\right) \psi$ be the direct sum of $X_{1}, X_{2}, \cdots, X_{n}$ equipped with the associated norm with $\psi$. Then we give the characterization of smoothness of $\left(X_{1} \oplus X_{2} \oplus \cdots \oplus X_{n}\right)_{\psi}$.

Mathematics subject classification (2000): 46B20, 52A21.

Key words and phrases: absolute normed space, smooth, uniformly smooth, norming functional.

\section{REFERENCES}

[1] B. BeAUZAMY, Introduction to Banach Spaces and their Geometry, 2nd ed., North-Holland, AmsterdamNew York-Oxford, 1985.

[2] R. BHATIA, Matrix Analysis, Springer, 1997.

[3] F. F. Bonsall and J. Duncan, Numerical Ranges, London Math. Soc. Lecture Note Series, Vol. 10, 1973.

[4] M. Kato, K.-S. Saito, T. Tamura, On $\psi$-direct sums of Banach spaces and convexity, J. Austral. Math. Soc., 75 (2003), 413-422.

[5] R. E. MegGinson, An Introduction to Banach Space Theory, Graduate Texts in Mathematics 183, Springer, 1998.

[6] K. Mitani, K.-S. SAIto, T. SuZUKI, Smoothness of absolute norms on $\mathbb{C}^{n}$, J. Convex. Anal., 10, (2003), 89-107.

[7] R. T. Rockafellar, Convex Analysis, Princeton University Press, Princeton, 1970.

[8] K.-S. SAITO, M. KaTO, Uniform convexity of $\psi$-direct sums of Banach spaces, J. Math. Anal. Appl., 277(2003), no. 1, 1-11.

[9] K.-S. SAITO, M. KATO AND Y. TAKAHASHI, Von Neumann-Jordan constant of absolute normes on $\mathbb{C}^{2}$, J. Math. Anal. Appl., 244 (2000), 515-532.

[10] K.-S. Saito, M. Kato, Y. TAKAHASHI, Absolute norms on $\mathbb{C}^{n}$, J. Math. Anal. Appl., 252 (2000), 879-905.

[11] Y. TAKAHASHI, M. KATO AND K.-S. SAITO, Strict convexity of absolute normes on $\mathbb{C}^{2}$ and direct sums of Banach spaces, J. Inequal. Appl., 7 (2002), 179-186. 